\title{
Exemples de résultats de la recherche obtenus sur les populations sauvages de salmonides de Bretagne et de Basse-Normandie et applicables à leur gestion Research on the wild salmonid populations in Brittany and Lower Normandy: possible applications in their management
}

\author{
J. L. Baglinière, G. Maisse, A. Nihouarn, J. P. Porcher et A. Richard
}

Volume 2, numéro 4, 1989

URI : https://id.erudit.org/iderudit/705059ar

DOI : https://doi.org/10.7202/705059ar

\section{Aller au sommaire du numéro}

\section{Éditeur(s)}

Université du Québec - INRS-Eau, Terre et Environnement (INRS-ETE)

ISSN

0992-7158 (imprimé)

1718-8598 (numérique)

Découvrir la revue

Citer cet article

Baglinière, J. L., Maisse, G., Nihouarn, A., Porcher, J. P. \& Richard, A. (1989).

Exemples de résultats de la recherche obtenus sur les populations sauvages de salmonides de Bretagne et de Basse-Normandie et applicables à leur gestion. Revue des sciences de l'eau / Journal of Water Science, 2(4), 859-874.

https://doi.org/10.7202/705059ar

\section{Résumé de l'article}

Ces dernières années, un ensemble de méthodes et techniques d'étude a été mis au point ou amélioré à partir de recherches réalisées sur les populations sauvages de salmonides. Dans un premier temps, ces nouvelles techniques peuvent être considérées comme des outils disponibles pour les gestionnaires; ce sont : la mise en place et l'homogénéisation de critères scalimétriques pour la détermination de l'âge des salmonidés migrateurs; la détermination du sexe par sérodiagnostic et à partir de critères morphométriques; l'estimation de la production en smolts de saumon à partir de l'habitat et des caractéristiques des peuplements automnaux. Dans un deuxième temps, l'application de certaines de ces méthodes dans le domaine écologique a permis d'obtenir des éléments essentiels pour la connaissance et/ou la gestion des populations sauvages de salmonides. 


\section{Exemples de résultats de la recherche obtenus sur les populations sauvages de salmonides de Bretagne et de Basse-Normandie et applicables à leur gestion}

Research on the wild salmonid populations in Brittany and Lower Normandy : possible applications in their management

J.L. BAGLINIERE(1), G. MAISSE(1), A. NIHOUARN(2), J.P. PORCHER(2), A. RICHARD (2)

RÉSUMÉ

Ces dernières années, un ensemble de méthodes et techniques d'étude a été mis au point ou amétioré à partir de recherches réalisées sur les populations sauvages de salmonides. Dans un premier temps, ces nouvelles techniques peuvent être considérées comme des outils disponibles pour les gestionnaires; ce sont : la mise en place et l'homogénéisation de critères scalimétriques pour la détermination de l'âge des salmonidés migrateurs ; La détermination du sexe par sérodiagnostic et à partir de critères morphométriques : l'estimation de la production en smolts de saumon à partir de l'habitat et des caractéristiques des peuplements automnaux. Dans un deuxième temps, l'application de certaines de ces méthodes dans le domaine écologique a permis d'obtenir des éléments essentiels pour la connaissance et/ou la gestion des populations sauvages de salmonides.

Mots clés : Salmonidés, populations sauvages, recherche, technique, gestion, France.

(1) Station de Physiologie et d'Ecologie des Poissons - 35042 Rennes Cedex, France.

(2) Délégation Régionale du Conseil Supérieur de la Pêche, 84, rue de Rennes, 35510 Cesson Sevigne, France. 


\section{SUMMARY}

During the last few years some new techniques for the study of wild populations of salmonids have been developed. The following procedures are intended to provide.

1) an improved method for determining age by scale-reading, particularly with migratory salmonids (At lantic salmon and sea trout). A standard zone for removing scales, the presence of three different zones on each scale (river, run-out, sea) and some criteria for scale interpretation have all been defined. These criteria are mainly concerned with the structure of the scale during the sea-life stage and reproduction. On the other hand, a spawning mark has been determined for both sedentary and migratory salmonids.

2) an means of sex determination. Two techniques have been proposed : - the first one is based on the detection by immunoagglutination of the serum vitellogenin of females in active gametogenesis. This method concerns females only; it does not allow a distinction to be made between non spermating males and immature salmonids. It is a very reliable technique which can be used in field conditions, but only by experienced researchers ;

- the second technique is based on the sexual dimorphism of the relative length of the upper $j$ aw bone. It is very reliable ( $8 \%$ to $12 \%$ error) and can also be applied in field conditions, but only for large fish (fork length > 40-45 cm).

3) a way of estimating Atlantic salmon smolt production. This technique is based on :

- the estimation of a $0+$ and $1+$ salmon population in the whole river from data of density obtained in sectors representative of the different types of habitat ;

- the presence of growth bimodality in a $0+$ salmon population in autumn, given the following hypothesis : one yearold smolts belong to the upper mode ;

- the estimation of mortatity for the whole winter season.

This technique has been used in the River $0 i r$ and compared with a smolt-trapping experiment conducted for two years. A comparison of the results shows that :

- the one year-old smolts belong to the upper mode of bimodality:

- when based on data on habitat characteristics, this technique can be used in field conditions to evaluate the smolts with sufficient accuracy.

All three techniques are immediately avai lable as useful methods for fisheries management. At a second stage, they can also serve in ecological research to advance knowledge on which to base further study and improved management of wild salmonid populations.

Key-words : Salmonids, wild populations, management, France. 
Depuis les années 1980, un ensemble de techniques et de méthodologies d'études a été mis au point ou amélioré à partir de résultat de recherches appliquées ou plus fondamentales réalisées sur les populations sauvages de salmonidés (Saumon atlantique, Salmo salar L. et Truite comune, Salmo trutta L.) de Bretagne et de Basse-Normandie. La mise au point et la validation de certaines de ces techniques ou l'amélioration et la standardisation des autres ont donné lieu à un certain nombre de publications (BAGLINIERE et al., 1981 ; BAGLINIERE, 1985 ; MAISSE et BAGLINIERE, 1986 ; RICHARD, 1986 ; BAGLINIERE et $a l ., 1988$ ).

Cet article presente d'une manière synthétique certaines de ces techniques ou méthodologies d'études acquises en abordant deux aspects :

- leurs intérêts et leurs applications pratiques pour la gestion des populations sauvages de salmonides en se restreignant volontairement à quelques exemples observés à 1 'échelon national ;

- leur utilisation au sein d'études plus approfondies permettant $a^{\prime}$ apporter de nouveaux éléments importants pour la connaissance et/ou la gestion de ces populations.

1 - LA DÉTERMination DE L'ÂGE

\section{1-1 La technique}

La dêtermination de 1 'âge chez les salmonidês se fait essentiellement à partir des écailles a nos latitudes (BAGLINIERE et LE LOUARN,. 1987). Si la méthode scalimétrique est utilisée depuis fort longtemps (DAHL, 1909 ; ROULE, 1920 ; JARVI et MENZIES, 1936 ; VIBERT, 1950 ; BACKIEL et SYCH, 1958) les critères d'interprétation des écailles n'avaient jamais fait encore 1 'objet de standardisation, ce qui reste utile notament dans la comparaison de caractéristiques de stock. Ainsi chez les salmonidés grands migrateurs à l'exception de la truite de lac, la méthode scalimétrique a été validée et standardisée suite à des réunions d'experts internationaux (ANONYME, 1984) et nationaux (BAGLINIERE, 1985 et 1987). Dans cette optique, il faut cependant souligner que le saumon atlantique reste 1 'espèce la plus étudiée. Cette standardisation de la méthode scalimétrique repose sur :

- le choix d'une zone de prélèvement optimale pour l'interprétation et la lecture des écailles. Cette zone a êté définie au niveau international depuis quatre ans chez le Saumon atlantique (ANONYME, 1984) et au niveau national très récement chez la truite de mer (OMBREDANE et RICHARD, 1989) (figure 1) ;

- la distinction entre trois phases de croissance de l'écaille caractéristiques d'un nigrateur amphibiotique à nos latitudes : rivière, transition et mer, ces trois phases ayant déjà été observêes par VIBERT (1950). Leur succession sur un grand axe de l'écaille est caractérisée 
par une augmentation de l'épaisseur et de l'espacement des circuli (BAGLINIERE et LE LOUARN, 1987);

- la mise en place de critères scalimétriques ; elle concerne essentiellement deux points :

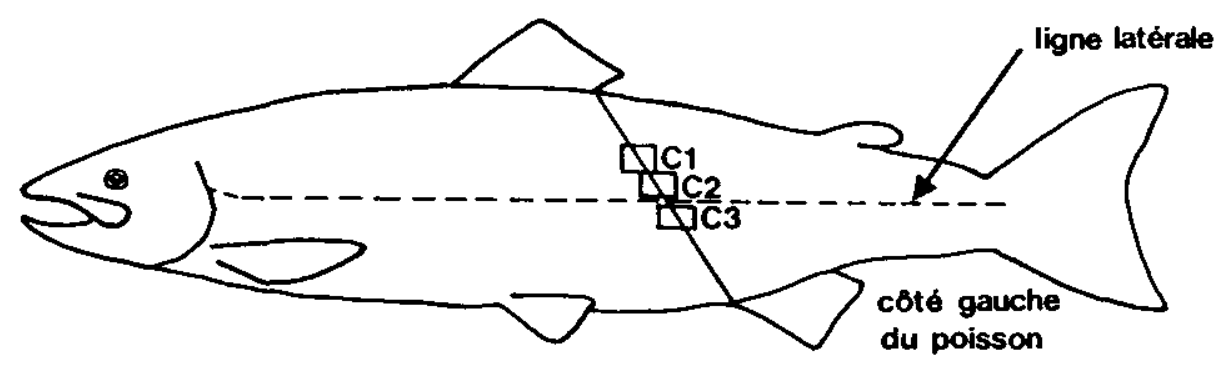

Figure 1. - Localisation du site de prélèvement d'écaille chez le saumon atlantique (C1 et C2) et chez la Truite de mer (C2 et C3) (BAGLINIERE, 1985 ; OMBREDANE et RICHARD, 1989).

Figure 1. - Standard zone of scales removing for At tantic Salmon (C1 and C2) and for Sea-Trout (C2 and C3) (BAGLINIERE, 1985 ; OMBREDANE and RICHARD, 1989).

\section{- la phase marine des grands migrateurs}

Chez le Saumon, des normes ont été établies au niveau international (ANONYME, 1984), vérifiées et homogénéisées au niveau national (BAGLINIERE, 1985). Chez cette espèce seul subsiste le problème de la détermination de l'âge de première remontée des individus migrant en fin d'année ou ayant fait un deuxième séjour en mer (résorption périphérique des champs latéraux et antérieur de l'écaille lors de la maturation sexuelle) (BAGLINIERE, 1985). Ce problème n'est pas à négliger puisque l'utilisation de plus en plus fréquente de stations de contrôle augmente le nombre de saumons êchantillonnés en fin d'année ; de plus les individus de deuxiłme remontée, bien que très peu nombreux, sont essentiellement des femelles (BAGLINIERE et al., 1987a) fournissant une contribution non négligeable au potentiel reproducteur annuel.

Chez la Truite de mer l'analyse scalimétrique des populations de Basse-Normandie a mis en évidence quatre types de poissons définis à partir de leur àge de ner à la première remontée (RICHARD, 1986). Cette analyse a êgalement révélé pour certaines populations l'existence de structures particulières de l'écaille en liaison avec des comportenents migratoixes variês des individus lors du stade post-smolt. La présence d'individus immatures remontant en rivière après avoir séjourné quelques mois en mer a ainsi été mise en évidence (figure 2). Les types de poisson et les structures particulières de l'écaille ont été confirmés par comparaison avec des populations de Truite de mer de Haute-Normandie et de Picardie lors d'une réunion du groupe de travail salmonidés migrateurs du Groupe Permanent de Concertation Poissons Amphihalins. Actuellement, une homogénéisation des normes de lecture doit être faite au niveau national et un premier examen des échantilions récoltés montre que les différents critères d'interprétation scalimétrique dégagés de l'étude des populations de Truite de mer de Basse-Normandie peuvent être considérés comme des éléments de référence. 


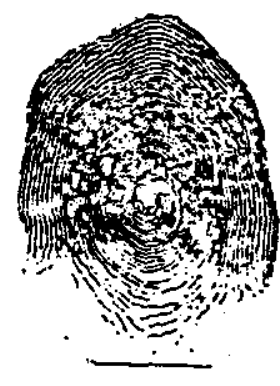

$+$

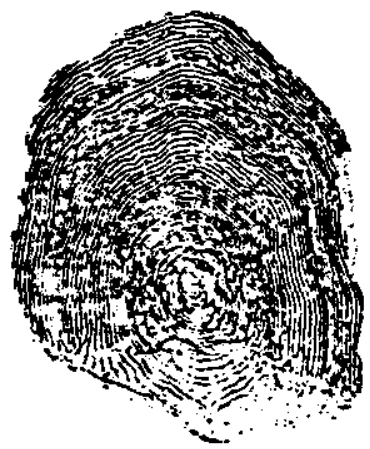

b

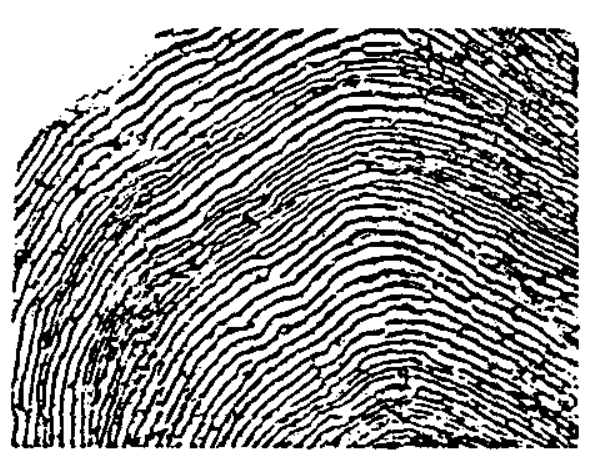

c

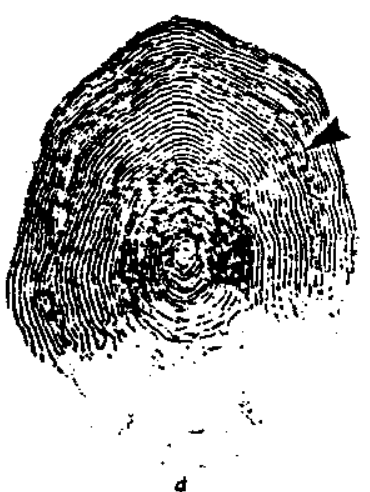

Figure 2. - Structure particuliẻre de l'écaille d'une truite de mer : double resserrement (D.R.) sur le premier hiver post smoltification (RICHARD, 1986).

a - ïinnock capturé à la montêe par piégeage et marqué le 01.08 .83 sur la rivière Touques (Calonne) (longueur fourche $5 L F)=307 \mathrm{mn}-$ poids $(P)=340 \mathrm{~g}-$ àge $=2+.0+1$

b - recapturé le 05.10 .84 à la montée sur la Touques (Calonne) $\left(L F=463 \mathrm{~mm}-\mathrm{P}=1040 \mathrm{~g}-\mathrm{Age}=2+.1+\mathrm{D} . \mathrm{R}_{\mathrm{S}}\right)$

c - détail du double resserrement

d - double resserrement en formation à la fin de l'hiver sur une écaille de truite de mer immâture capturêe à la ligne dans l'estuaire de la Touques le 09.03.84 (LF $=340 \mathrm{~mm}$ $\mathrm{P}=365 \mathrm{~g}$ - poux de mer - Age = $1+.1 \mathrm{D} . \mathrm{R}$.

— remontée estivale (légère érosion)

Figure 2. - Particular structure of Sea Trout scale : äouble narrow-spaced circuli bands (dark bands $=D . B$.$) after the$ first growth season in the sea.

a - finnock trapped during upstream migration and tagged the 83.08.01 in Touques river (Calonne) (fork length (LF) $=$ $307 \mathrm{mon}-$ Weight $(W)=340 \mathrm{~g}-$ Age $=2+.0+$ )

$b$-recaptured during upstream migration the 84.10 .05 in Touques river (Calonne) $(L F=463 \mathrm{~mm}-P=1040 \mathrm{~g}-$ Age $=2+.1+$ D.B.)

$c$ - structure of double narrow-spaced circuli bands

$d$ - beginning of structure of double darks bands on a scale of immature sea-trout caught by rod in estuary of Touques river the $84.03 .09(\mathrm{LF}=340 \mathrm{~mm}-P=365 \mathrm{~g}$ - sea lice Age $=1+.1$ D. B.

— sumer upstream migration (light erosion of scale) 


\section{- la marque de frai ou de ponte}

Cette marque de ponte observée par JARVI et MENZIES (1936) et BACKIEL et $\mathrm{SYCH}$ (1958) a été ređéfinie plus récemunt et plus précisêment à la fois pour les migrateurs holobiotiques et amphibiotiques (ANONYME, 1984 ; BAGLINIERE, 1985 ; RICHARD, 1986 ; MAISSE et al., 1987). La première reproduction peut avoir lieu chez les espèces grandes migratrices avant leur descente en mer ou en lac (BAGLINIERE et MAISSE, 1985). Enfin lors de la maturation sexuelle, l'érosion de l'écaille ne se fait pas de la même manière chez le Saumon et la Truite. Chez cette dernière, c'est la partie postérieure non recouverte de l'écaille qui est la plus résorbēe.

Pour dêterminer 1'âge des migrateurs holobiotiques ou le temps de séjour en eau douce des juvéniles des espèces amphibiotiques, aucune norme n'a été établie, mise a part la définition de bande hivernale (ligne d'arrêt de croissance, annulus) (ANONYME, 1984 ; BAGLINIERE et LE LOUARN, 1987). Seuls des exemples typiques et atypiques d'écailles de poissons d'áge différent ont été présentés chez le saunon atlantique (ANONYME, 1984 ; BAGLINIERE, 1985) et des critères d'aide à la dêtermination ont êtê fournis (BAGLINIERE, ibidem).

\section{1-2 Les intêrêts}

Ia nise en place et la normalisation de critères scalimetriques permet de rendre plus fiable la méthode de determination de l'age à partir des écailles. Par ailleurs elle renforce les caractères (facilité de mise en oeuvre et d'interprétation) de cette méthode parfaitement adaptée aux études de dynamique de population contrairement à d'autres (otolimétrie) qui nécessitent le sacrifice du poisson (BAGLINIERE et al., 1986).

Outre l'âge individuel et celui de première maturité, l'utilisation de cette méthode permet de connaitre le temps de renouvellement, la structure démographique d'un stock et la composition en àge des captures réalisêes sur un cours d'eau. D'une manière annexe, l'emploi des écailles permet également d'analyser le taux de croissance d'une population et de différencier nettement le Saumon atlantique de la Truite de mer en prenant en compte la dimension, la forme et la structure de 1 'écaille lors de la première année en mer (BAGLINIERE.et LE LOUARN, 1987).

Cette normalisation et cette homogénéisation de l'interprétation des écailles a permis de réaliser une étude sur les populations de saumon en France à partir de données récoltées sur l'ensemble des rivières depuis 1968 (relecture ou lecture des écailles de tous les poissons capturés à partir de cette époque) (PREVOST, 1987 ; BAGLINIERE et PREVOST, 1987). Cette étude a donné une vision synthétique et comparative des caractéristiques de populations de saumon en montrant l'importance de l'outil scalimétrique dans la connaissance des stocks. Il en ressort principalement :

- un temps de renouvellement très court de 2 à 4 ans ;

- la présence de castillons (1+ de mer) sur l'ensemble des rivières confirmant les observations de BENARDEAU (1905) et de ROULE (1920). Ce groupe d'âge de mer apparait important dans les stocks des cours d'eau du Massif Armoricain et de certaines rivières du sud Ouest ; 
- la relation entre la régularité et la puissance hydraulique des cours d'eau et la taille et l'âge de mer à la première remontée des adultes ;

- l'existence d'unités biogéographiques par région (Bretagne-Nord. Bretagne-Sud, Basse-Normandie) ou par grand bassin hydrographique (Adour-Nivelle et Loire Alliex) avec la prêsence de deux cours d'eau bien particuliers : l'Allier et le Gave d'Oloron ;

- la difficulté à discriminer des stocks proches géographiquement à partir de variables biomêtriques et scalimétriques.

\section{1-3 Applications pratiques}

Dans le cas du saumon l'emploi de la scalimétrie a permis de caractériser d'une manière plus précise que la simple utilisation de la longueur (analyse de l'histograme de fréquence de taille) ou du poids, les diffërentes composantes des stocks français. Ainsi les statistiques de captures sont maintenant présentées au niveau national en distinguant les saumons ( 2 ans et plus de mer) des castillons (2+ an de ner), cette distinction étant une des recommandations faites par le conseil International pour l'Exploration de la Mer (CIEM). Par ailleurs les normes scalimétriques établies pour l'âge marín ont montré l'existence de castillons tardifs remontant en.fin d'année et échappant à tout type d'exploitation en eau douce. Ces poissons ont des caractéristiques morphométriques qui se rapprochent de celles des saumons de deux ans de mer remontant en début d'année. De plus, le rapport des sexes de ces castillons taxdifs est nettement plus en faveur des femelles que chez les individus précoces (BAGLINIERE et al., 1987a). Ainsi la mise en évidence de l'importance des castillons dans certains stocks et la présence de renontées tardives a permis de proposer une augmentation des quotas de captures de saumon dans les rivières du Massif Armoricain à partir du premier juin.

Pour la truite de mer, la caractérisation des populations du dếpartement du Calvados (1980 à 1985), notament à partir des études scalimétriques (âge de prenière maturité, gêniteurs survivant à la fraie) a permis la mise en place d'une rẻglementation de pêche adaptée aux stocks: périodes d'ouvertures calées sur les rythmes de remontées, taille légale de capture et protection des stades sensibles.

\section{2 - La détermination du SEXE}

\section{2-1 Les techniques}

Les caractères sexuels secondaires affectant la morphologie des sa1monides $n$ 'apparaissent que tardivement et ne sont pas toujours suffisanment marqués, en particulier chez les poissons de première reproduction (LE BAIL, 1981). La détermination du sexe chez les salmonidés maintenus vivants $n$ 'est donc possible que durant la période de reproduction. En dehors de cette période, le sexage nécessite le sacrifice des animaux. Deux techniques nouvelles ont été mises au point : 
- Détermination du sexe par prélèvement sanguin

LE BAIL et BRETON (1981) ont mis au point une technique d'imunoagglutination permettant la mise en évidence rapide de niveaux élevés de vitellogénine plasmatique, caractéxistique des fenelles en gamétogênèse active. Cette technique permet donc d'individualiser l'élément femelle d'une population de saumon dès le mois de mars et à partir du mois d'avril pour la truite. Après sa mise en oeuvre pratique réalisée par LE BAIL et al. (1981), cette méthode a été utilisêe pour de nombreuses études concernant les populations sauvages de salmonides (BAGLINIERE et al., 1981 ; MOUTOUNET-ARRIBE, 1981 ; PROUZET et JEZEQUEL, 1983 ; PROUZET et al., 1984 ; MAISSE et BAGLINIERE, 1986 ; MAISSE et al., 1986 ; RICHARD, 1986 ; MAISSE et al., 1987). La fiabilité de cette méthode est totale (LE BAIL, 1981 ; LE BAIL et BRETON, 1981 ; MAISSE et BAGLINIERE, 1986).

- Détermination du sexe à partir de critères morphométriques

La détermination du sexe par prélèvement sanguin, si elle ne nécessite pas l'autopsie du poisson, n'en reste pas moins une méthode d'une utilisation réservée à un personnel entraîné (prise de sang). Ainsi il s'est révélé nécessaire de mettre au point une technique plus facilement adaptêe aux conditions de terrain.

LE BAIL (1981) a mis en évidence en particulier chez la truite de mer de la rivière Touques (Basse-Normandie) un dimorphisme sexuel de la longueur de la mâchoire supérieure, d'autant plus net que les poissons sont âgés et/ou proches de la reproduction. S'inspirant de ces résultats, des adultes de saumon atlantique ont été sexés en été à partir de la longueur relative de la mãchoire supérieure avec une marge d'erreur faible sur une rivière écossaise $(7$ d'erreur : MAISSE et BAGLINIERE, 1986) et sur quatre rivières québécoises (12 d'erreur : MAISSE et al., 1988) (figure 3). Le même type d'étude a été réalisê sur les populations de truite de mer de Basse-Normandie depuis le printemps jusqu'à l'automne. Elle montre également la possibilité de sexer les adultes de truite de mer (mai à juillet) à partir de ces dimorphisme sexuel avec une marge d'erreur faible $\left(\begin{array}{ll}10 & \text { z }\end{array}\right)$ à condition que la taille du poisson soit supérieure à $40-45 \mathrm{~cm}$ (RICHARD, 1986).

\section{2-2 Intẽrêts}

La comparaison des différentes techniques de détermination du sexe montre que celle utilisant le dimorphisme de la mâchoire supérieure peut être recommandée au praticien en raison de sa marge d'erreur faible, de sa facilité d'exécution et de son objectivité. Les conditions restrictives d'application (longueur fourche $>40-45 \mathrm{~cm}$ chez la truite) excluent la possibilité d'utilisation de cette méthode chez la truite pour les populations sédentaires et en partie pour celles du groupe d'âge ot de mer. Par ailleurs l'emploi de cette méthode nécessite la mise en place d'un abaque de référence pour chaque stock et à différentes périodes de l'année (mars à août). Elle nêcessite également la prise en compte des composantes castillons-individus de plusieurs hivers de mer chez le saumon et celle des of de mer, poissons vierges et poissons ayant déjă frayê chez la truite de mer. 


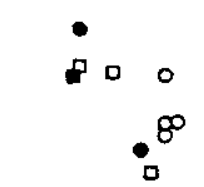

$\because \because 0^{\circ} 0^{\circ}$

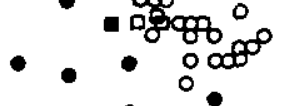

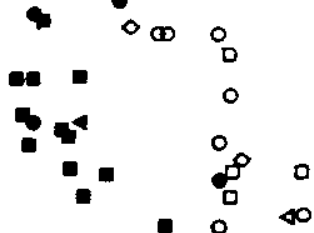
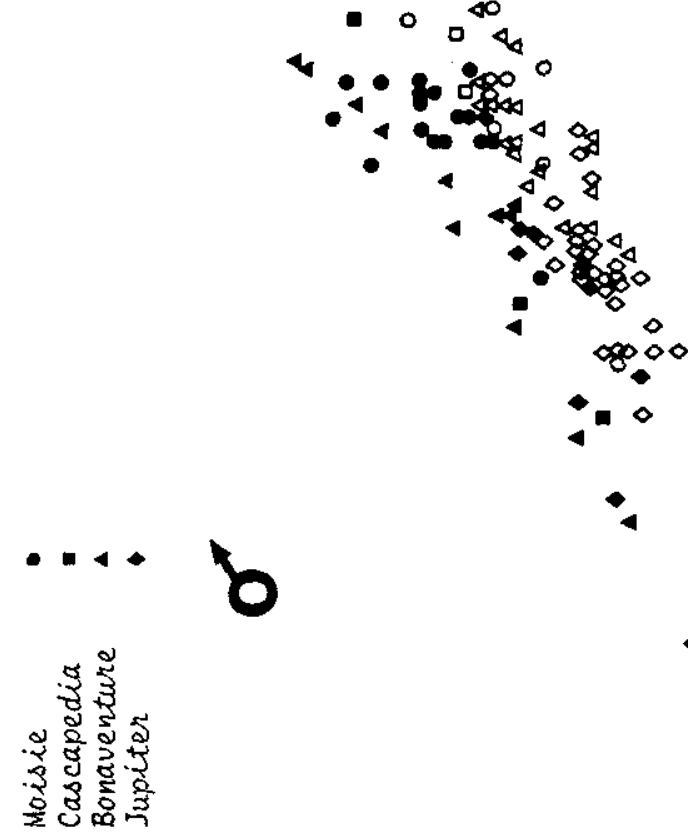

$\dot{\propto} \dot{x} \dot{x} \dot{x}$

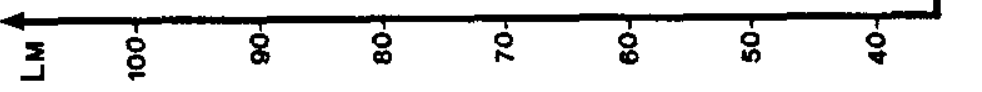

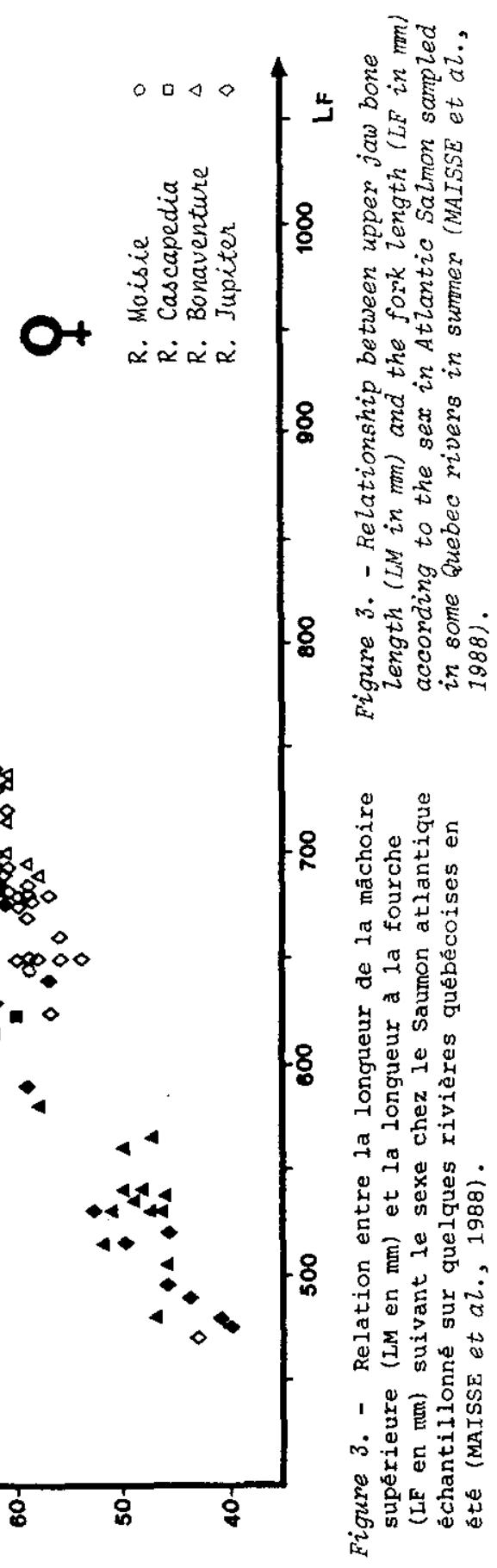




\section{2-3 Applications pratiques}

Cette technique morphométrique de détermination du sexe ainsi que celle effectuée à partir d'un prélèvement sanguin permet non seulement de connaitre le rapport des sexes dans des limites raisonnables de fiabilité mais également de déterminer le potentiel reproducteur, paramètre indispensable pour les études de dynamiques de population et la gestion des stocks. Ceci nécessite cependant l'obtention d'une relation longueur fourche-fécondité (nombre d'ovules) pour la population étudiée (PROUZET et al., 1984). L'utilisation de cette méthode a permis d'estimer le potentiel reproducteur sur deux rivières à saumon : Elorn, Bretagne (BAGLINIERE et DUMAS, 1988) et Oir, Basse-Normandie (BAGIINIERE, MAISSE, NIHOUARN et PORCHER, données non publiées). La connaissance de ce paramètre permet alors d'envisager le prélèvement de géniteurs en tenant compte de la capacité d'accueil du milieu. Ceci a été réalisé sur la rivière oir où au cours de trois années d'étude, les valeurs estimées du potentiel reproducteur de saumon atlantique sont apparues disproportionnées (1055 à 1289 oeufs/100 $\mathrm{m}^{2}$ ) par rapport à celles de la production en smolts ( 1 à 3 individus/100 $\mathrm{m}^{2}$ ) lorsque 1'on considère les normes ëtablies par syMoNs (1979). Il semble donc qu'une ponction d'oeufs sur une partie du stock de femelles adultes nigrantes soit envisageable sans aucune modification de la production naturelle en juvéniles de la rivière. Ainsi on peut réaliser une production en smolts à partir de ce surplus d'oeufs qui pourra se surajouter à la production naturelle en n'étant pas au détriment de celle-ci.

La dêtermination du sexe par prélèvement sanguin a permis d'entreprendre une étude approfondie de la dynamique de population de la truite commune sur un petit ruisseau. Elle a rendu possible la caractérisation de la population de géniteurs sédentaires du ruisseau (MAISSE et al., 1987) à laquelle s'ajoutait celle de géniteurs migrants provenant de la rivière principale en période de reproduction (BAGLINIERE et al., $1987 b)$. Le cycle biologique ainsi observé se caractérise par la séquence suivante (BAGLINIERE et al., 1989).

géniteurs de la rivière principale $\longrightarrow$ géniteurs du ruisseau $\longrightarrow$ juvéniles dévalant du ruisseau vers la rivière principale.

Mais l'aspect essentiel du point de vue pratique, qui ressort de cette étude est la démonstration de l'importance de la tête de bassin de ce ruisseau frayère qui produit à elle seule 84 des juvéniles migrants. La nécessité de la protection de cette zone est évidente et vitale pour le maintien d'une population de truites adultes exploitables dans la rivière.

3 - ESTIMATION DE LA PRODUCTION EN SHOLTS DE SAUMON ATLAIITIQUE

\section{3-1 La technique}

Pratiquée en France surtout depuis les années 1950 (VIBERT, 1950), le piégeage est resté longtemps la seule méthode utilisée pour estimer 
la production en smolts de saumon atlantique des rivièxes françaises. Cette dernière se caractérise par la présence de deux classes d'âge 1+ et 2+ (BAGLINIERE, 1976 ; BOUSQUET, 1979; LE TEUFF, 1985 ; FOURNEL et al., 1987 ; PREVOST, 1987 ; TELLIER, 1987).

Une autre méthode a été récemment mise au point par BAGLINIERE et ChAMPIGNEULLE (1986) pour estiner la production en smolts de saumon sur un réseau hydrographique. Elle est basée sur la caractérisation des peuplements automnaux de tacons en fonction des caractéristiques de 1 'habitat et se décompose en trois points :

- estimation des densités automnales des peuplements en tacons o+ et $1+$ dans des secteurs représentatifs des différents habitats. En raison de préférenda physiques bien marqués chez les jeunes saumons pour les zones courantes peu profondes (BAGLINIERE et CHAMPIGNEULLE, 1982 ; BAGLINIERE et ARRIBE-MOUTOUNET, 1985), cette estimation peut être réalisẻe sur la totalité du cours d'eau après cartographie. Le milieu profond $n^{\prime a}$ pas été pris en compte en raison de la quasi absence de juverniles ;

- analyse de 1'histograme de fréquence de taille des tacons $0+$ : Ia mise en évidence d'une éventuelle bimodalité est rêalisée par la méthode de BATTACHARYA (LAURENT et MOREAU, 1973). Par analogie avec ce qui a été décrit par THORPE et al. (1980), les individus du mode haut sont supposés se smoltifier au printemps suivant, ceux du mode bas restant au moins une année supplémentaire en rivière;

- estimation des mortalités hivernales suivant l'âge et la taille par marquage.

Cette méthode d'estination a été mise en pratique sur la rivière oir (affluent de la Sélune, Basse-Normandie) et comparée avec celle du piégeage sux deux années. Les résultats de cette étude montrent que (BAGLINIERE et al., i988) :

- les estimations en smolts réalisées pax les deux mêthodes sont très proches avec cependant des différences plus ou moins importantes selon l'année (tableau 1);

- les smolts 1+ appartiennent bien au groupe d'individus à forte croissance présents dans la population de saunons ot en autome (figure 4).

\section{3-2 Intērêts}

Cette méthode d'estination en smolts fondèe sur la caractérisation des peuplements autornaux de saumons en fonction des caractéristiques de l'habitat permet d'abord d'obtenir des valeurs de production dans des limites raisonnables de fiabilité. Par ailleurs, que les objectifs se situent au niveau de la gestion ou de la connaissance des stocks, il importe souvent plus de cerner les variations annuelles de production que les valeurs absolues. En outre l'estination du nonbre de juvéniles rigrants rend possible la prévision du nombre d'adultes en relation avec les caractéristiques du stocks et du cours d'eau. Ensuite cette méthode présente l'avantage de limitex les interventions sur le terrain contrairement au piégeage qui nécessite : 
- des installations coûteuses pour lesquelles un suivi matériel et humain doit être mis en place à long terme (durée de la dévalaison égale à trois mois),

- une manipulation des poissons, particulièrement fragiles au stade smolt, afin de connaitre l'efficacité du piège (méthode de capturerecapture).

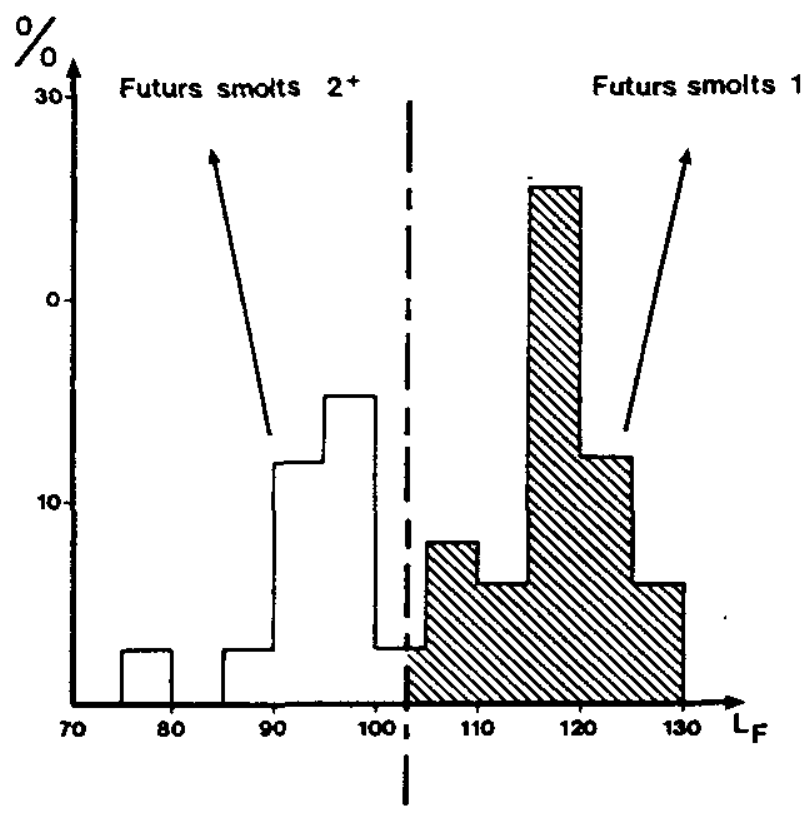

Figure 4. - Histogramme de fréquence de tailie ( ge, $L F$ en mu = Iongueur fourche) de la population de saumon or échantillonnée sur un secteur de l'oir en automne 1985. ----- droite de séparation de la bimodalité.

Figure 4. - Distribution of size frequency $(\%$ = percentage, $L F$ en $m$ = fork length) in of salmon population sampled in a sector of oir river during 1985 autumn.

-.....- limit size between the two modes.

Cette méthode peut également être utilisée pour calculer la potentialité d'un milieu permettant de chiffrer les pertes dans le cas d'êtudes d'impact des activités humaines ou les besoins dans le cadre d'opérations de restauration. Dans tous les cas cela permet d'orienter correctement le processus de réhabilitation du milieu (RICHARD, 1988). Ces deux derniers aspects peuvent concerner également la truite comune, dans la mesure où une fourchette des valeurs de la capacité d'accueil du milieu a pu être établie pour les deux espèces de salmonides en fonction des caractéristiques de l'habitat, des conditions climatiques annuelles et' de 1 'âge du poisson (BAGLINIERE et CHAMPIGNEULLE, 1982 ; BAGLINIERE et CHAMPIGNEULLE, 1986). 
Tableau 1. - Estination de la production en smolts de saumon atlantique sur la rivière Oir en 1986 et 1987.

Table 1. - Estimation of atlantic salmon smolts production in Oir river in 1986 and 1987.

\begin{tabular}{|c|c|c|c|c|c|c|c|}
\hline \multirow[t]{2}{*}{ Année } & \multirow[t]{2}{*}{ Méthode } & \multicolumn{4}{|c|}{$\begin{array}{c}\text { Nombre de smolts } \\
\text { estimé }\end{array}$} & \multicolumn{2}{|c|}{ Total } \\
\hline & & $1+$ & & & $2+$ & & \\
\hline \multirow[b]{2}{*}{1} & $\begin{array}{l}\text { Caractérisation de I'ha- } \\
\text { bitat par tronçon }\end{array}$ & $1019 \pm$ & +298 & 182 & \pm 33 & $2201 \pm$ & 270 \\
\hline & & & & & & & \\
\hline \multirow{5}{*}{$\begin{array}{l}9 \\
8 \\
6\end{array}$} & Caractérisation de 1'ha- & $924 \pm$ & \pm 180 & 176 & \pm 22 & $1100 \pm$ & 147 \\
\hline & bitat sur 1 'ensemble du & & & & & & \\
\hline & cours d'eau & & & & & & \\
\hline & Piégeage & $1.270 \pm$ & \pm 181 & 55 & \pm 8 & $1325 \pm$ & 189 \\
\hline & $\begin{array}{l}\text { Caractérisation de l'ha- } \\
\text { bitat par tronçon }\end{array}$ & $397 \pm$ & \pm 185 & 343 & \pm 48 & $740 \pm$ & 158 \\
\hline \multirow{5}{*}{$\begin{array}{l}1 \\
9 \\
8 \\
7\end{array}$} & Caractërisation de l'ha- & $308 \pm$ & \pm 103 & 295 & \pm 26 & $503 \pm$ & 95 \\
\hline & bitat sur l'ensemble du & & & & & & \\
\hline & cours d'eau & & & & & & \\
\hline & $\begin{array}{l}\text { Inventaire de l'ensemble } \\
\text { du cours d'eau }\end{array}$ & $191 \pm$ & 11 & 232 & \pm & $423 \pm$ & 9 \\
\hline & Piégeage & $199 \pm$ & $\pm \quad 37$ & 180 & \pm 33 & $379 \pm$ & 70 \\
\hline
\end{tabular}

\section{COHCLUSIONS}

Les différentes techniques d'étude exposées montrent certaines imperfections et limites dans leur chanp d'application liées à I'état actuel des connaissances. Ce sont :

- en scalimétrie, la méconnaissance de l'évolution de la croissance et de la structure de l'écaille avec les facteurs de l'environnement,

- pour la déternination du sexe, la difficulté d'utilisation courante de la technique par prélèvement sanguin alors que celle de la mesure de la mâchoire supérieure est limitée à l'étude de gros individus,

- pour l'estination de la production en smolts, 1'absence d'une fourchette de valeurs des mortalités hivernales.

Lorsque l'on considère le domaine de la gestion des populations sauvages de salmonidés, les imperfections au plan méthodologique de ces techniques apparaissent nineures comparées à certaines linites du chamo d'application. En dépit de ces résexves, ces techniques d'êtude présentent un intērét indéniable dans le domaine de la gestion pour quatre 
raisons : disponibilité, mise en ouvre pratique aisée, facilité d'emploi et ordre de grandeur d'une précision très suffisante pour orienter valablement les décisions en matière de gestion.

De plus, l'utilisation de ces techniques doit permettre d'honogénéiser la récolte et l'obtention de résultats sur des séries pluriannuelles. De telles séries font actueliement défaut et sont indispensables pour intégrer les tendances à long terme en les différenciant de simples variations annuelles dans la gestion des populations naturelles.

Par contre, les imperfections de ces techniques apparaissent plus limitantes dans le champ d'application de la recherche et nécessitent des études complémentaires.

Dans le cas présent, les techniques ou les méthodologies d'étude présentées issues de résultats de recherches débouchent sur de réelles applications pratiques dans la gestion des stocks de salmonides. Cependant la liaison réciproque entre les deux domaines peut s'avêrer quelquefois difficile dans le cas:

- d'un résultat de recherche posant des problènes d'application : par exemple la mise en évidence d'une taille de première maturité chez la truite différente suivant 1 'ordre de drainage des milieux d'un même bassin (MAISSE et BAGIINIERE, données non publiêes) ;

- d'un manque de connaissances scientifiques ne permettant pas de rëpondre à un problème posé par un organisme décideur : par exemple, maintien ou non de la fermeture de la pêche aux salmonidés migrateurs dans l'estuaire de la Gironde (BAGLINIERE, 1987).

Au-delà de ces considérations il n'en reste pas moins que ces deux grands ensembles Recherche et Gestion des populations de salmonides s'interpénètrent difficilement en raison de problématiques propres parallèles. Il en est pour preuve que la gestion de ces populations n'a pas nëcessitê un cextain niveau de connaissances scientifiques pour exister. Cependant, actuellement, un effort mutuel semble se réaliser afin de prendre en compte les apports et les demandes de chacun des deux ensembles.

\section{RÉFミRENCES BIBL IOGRAPHIQUES}

ANONYME (1984). Atlantic Salmon scale reading. Report of Atlantic scale reading workshop, aberdeen, Scotland 2328 april, 1984. ICES 1 vol., 17 p.

BACKIEL T. SYCH R. (I958). Scales resorption and spawning marks in sea trout (Salmo trutta $\mathrm{L}$.) Erom polish waters. Roc. Nauk Rot., 73 ser. B : 119-148.

BAGLINIERE J.L. (I976). Etude des populations de Saunon atlantique (Salmo salar L. 1766) en Bretagne-BasseNormandie. 1 - Caractéristiques des smolts de la rivière Ellé. Ann. Eycrobiol., 7 : 141-158.
BAGLINIERE J.L. (1985). La déterminazion de 1'âge par scalinétrie chez le sation atlantique dans son aire de répartition méridionale : utilisation pratique et difficultés de ia méthode. Eü:. En. Peche Piscic., 298 : 69-105.

BAGLINIERE J.L. (1987). Compte rendu de la reurion du Groupe de Travail salmoniđés migrateurs du GPC poìssons AmphinaIins. Station de Physiologie et d'Ecologie des Poissons, INRA Rennes, $6 \mathrm{p}$. 
BAGLINIERE J.L., ARRIBE-MOUTOUNET 1985) . Microrépartition des populations de Truite cormune (Salmo trutta 1.), de juvénile de Saumon atlantique ISalmo salar t.) et des autres especes pressontes dans la partie haute du scorff (Bretagne). Hydrobiologia, 120 : 229239.

BAGLINIERE J.L., CHAMPIGNEULLE A. (1982). Densité des populations de Truite conmune (Salmo trutta $\mathrm{L}$.) et de juvéniles de Saumon atlantique (Salmo salar L.) sur le cours principal du Scorff (Bretagne) : preferendums physiques et variations annuelles (1976-1980). Acta becologie Decol. Applice., 3 : 241-256.

BAGLINIERE J.L., CHAMPIGNEULLE A. (1986) . Populations estimates of juvenile $\lambda t$ lantic Salmon (Salmo salar) as indices of smolts production in the River Scorff, Brittany. J. Fish. Biol., 29 : 467-482.

BAGLINIERE J.L., DUMAS J. (1988). Rêintroductions, restaurations et soutiens dc populations de Saumon atlantique (Salmo salar L.) en France. INRA, Dep. Hydrobiol. Bull. Sci. Techn., 24, 32 p.

BAGLINIERE J.L., LEBAIL P.Y., MAISSE G. (1981). Détection des femelles de salmonidés en vitellogênése. 2 - un exemple d'application : recensement dans la population de truite comone ISalmo trutta L.) d'une rivière de Bretagne-Sud, le scorff. Bull. Fr. Fiscic., 283: 89-95.

BAGLINIERE J.L., LECLERC G., RICIIARD $A$. (1986). Comparaison entre 1 "âqe et 1a croissance déterminés par scalimétrie et otolimetrie chez la Truite de mer (Salmo trutta L.). Bull. Fr. Pêche Piscic., 301:56-66.

BAGLINIERE J.L., LE LOUARN $h$. (1987). Caractéristiques scalimétriques des principales especces de poisson d'eau douce en France. Bull. Fr. Pêche Piscic., $306: 1-39$.

BAGLINIERE J.L., MAISSE G. (1985). Precocious maturation and smoltification in wild atlantic salmon in the armoricain Massif, France. Aquaculture, $45:(1-4): 249-263$.

BAGLINIERE J.L., MAISSE G., LE BAIL P.Y., NIHOUARN A. (1989). Population dynamics of brown Trout (Salmo itutta L.) in a tributary in Bxittany (France) : Spawning and juveniles. I. Fish. Biol., 34 : 97-110.

BAGLINIERE J.L., MAISSE G., LEBAIL P.Y., PREVOST E. (1987b). Dynanique de population de truite commune (Salmo irutta L.) d'un ruisseau breton (France) : ies géniteurs migrants. Acta Decologia decol. Applic., $8: 201-215$.
BAGLINIERE J.L., MAISSE G., NIHOUARN A., PORCHER J.P., LE GLOANEC P. (1988). Comparaison de deux methodes d'estimation de la production naturelle de smolts de soumon atlantique (Salmo salar 1. .). bull. F'r. Pêche Piscic, 300 : 24-34.

BAGLINIERE J.L., PREVOST E. (1987). Analyse comparative et discrininante des populations de saumon atlantique partir de critères d'áge scalimetrique. Rapport final, accord cadre INRA-IFRFMER 1985. Station de Physiologie et d'Ecologie des Poissons, INRA Rennes, $7 \mathrm{p}$.

BAGLINIERE J.L., PROUZET P., PORCHER J.P., NIIOUARN $A .$, MAISSF, G. (1987a). Caractéristiques gênérales des populations de Saumon atlantique (Salmo salar L.) des rivières du Massif armoricain. In "La restauration des rivieres a saumon" $\mathrm{Col}$ loque franco-québecois 28 mai-1er juin 1985. Hydrobiologie et Aquaculture. Ed. M. Thihault et R. Billard, 23-37.

BENARDEAU F. (1905). Pêche et reproduction du sawmon en loire. Ed. BergerLevrault et Cie, Paris-Nancy, 1 vol.. $51 \mathrm{p}$.

BOUSQuET B. (1979). Biologie et migration des smolts de-saumon atlantique ISalmo salar L.) dans les bassins loire-Allier et Adour-Gave d'oloron. Thèse 3ème cycle. Sci. Techn. Prod. Anim. Inst. Nation. Polytechn., Toulouse, $67 \mathrm{p}$.

DAHL R. (1909). The assessment of age and growth in fish. Int. Revue der gesamt. llydrobiol. und lydrogr., 2 : 758-769.

FOURNEL F., EUZENAT G., FAGARD J.L. (1987). Rivieres a truites de mer et a saumons de Haute-Normandie. Réalités et perspectives. In : M. Thibault et R. Billard, (Eds.). "La restauration des rivières d saumon". INRA Paris, 315-334.

JARVI T.H., menzIES N.J.M. (1936). The interpretation of the zones on scales of salmon, sea trout and brown trout. Cons. Perm. Expl. Mer XCVII, 1 vol., 63 p.

LAURENT M. , MOREAU G. (1973). Influence des facteurs écologiques sur le coefficient de condition $d^{\prime}$ un téleostéen (Cottus gobio L.). Ann. Hydrobiol., 4 : 211-228.

LEBAIL P.Y, (1981). Identification du bexe en fonction de l'etait de maturité chex les poissons. These de Docteur Ingênieur, Ecole Nat. Sup. Agronomie Rennes, I Vol.., $71 \mathrm{p}$.

LEBAIL P.X., BRETON B, (1981). Rapid determination of the sex of puberal salmonid fish by a technique of immunoagglutination. Aquaculture, 22 : 367-375. 
LEBAIL P.Y., MAISSE G., BRETON $\equiv$. (1981). Détection des femelles de salno:idés en vitellogénése. I - Description Ẽ la méthode et nise en oeuvre pratique. Bull. $F^{2}$. Piscic., 283 : 79-88.

LE TEUFE P. (1985). Premiêres cinservations sur les populations de saitonidès, Truite comune (Saimo trutta i.) et Saumon atlantique (Salmo salar I.) Eans l'oir (Bassin de la Sellune, Manche). ICaractéristiques de la dévalaisen. 2 Estimation de la production en :-ivéniles. Diplóme d'Agrononie Approfondie, Section halieutique. Ecole Nationale Sunźrieure Agronomique de Rennes, 1 vol.., jo p.

MAISSE G., BAGLINIERE J.L. (1966). Le sexage morphologique du Saumon $a=l a n t i-$ que (Salmo salar). Bulz. Er. Pềie Piscic., $300: 13-18$.

MAISSE G., BAGLINIERE J.L., BREZON B. FOSTIER A. (1986). Etude de la $\equiv$ :ologie des finnocks (Salmo trutta) de is rivière Touques, Calvados. Rapport fina:. Convention CSP-INRA ( $n^{\circ}$ 2565B) Staticr de Physiologie et d'Ecologie des pcissons, INRA Rennes, $5 \mathrm{p}$.

MAISSE G., BAGLINIERE J.L., LANERY G., CARON F., ROULEAU A. (1988). L'identification externe du sexe chez le saumon atlantique (Salmo salar L.). Câ. $J$. 200l., 66 : 2312-2315.

MAISSE G., BAGLINIERE J.L., LESÀ-Z P.Y. (1987). Dynamique de population je truite commune (Salmo trutita) d'un ruisseau breton (France) : les géniteurs sécentaires. Hydrobiologia, 148 : 123-130.

MOUTOUNET-ARRIBE D. (1981). Etude du Saumon atlantique (Salmo salar :.) en Bretagne-Sud. 1 - Analyse des captures par péche à la ligne sur le Scorš́, l'Ellé et le Blavet. 2 - Répartizion spatio-temporelle des juvéniles je saumons sur un secteur du Scorff. Pzéservation et Aménagement du Milieu Nasurel. Ecole Nationale Supérieure Agroronique de Rennes, i vol., 33 p.

OMBREDANE D., RICHARD A. (1990). Jétermination de la zone optimale de zzélèvements d'écailles chez les juvéni ies de truite de mer (Salmo trutta L.). Iull. Fr. Pêche Piscic. (sous presse).

PREVOST E. (1987). Les populati-1s de Saumon atlantique (Salmo salar :.) en France : Description; Relation . vec les caracterristiques des mivierez; Essai de Discrimination. Thèse $=50$. Ingénieur Sci. Agron. ENSA Renres, 1 vol., 103 pp.
PROUZET P., JEZEQ̨UEL M. (1983). Caractéristiques des populations de saunon atlantique (Salmo salar L.) capturées a la ligne sur l'Elorn (rivière ce Bretagne Nord) durant la période 19741981. Bull. Fr. Piscic., 289 : 94-111.

PROUZET P., LEBAIL P.Y., HEYDORFF M. (1984). Sex ratio and potential fecundity of Atlantic salmon (Salmo salar L.) caught by anglers on the Elorn River (Northern Brittany, France) during 1979 and 1980. Fish Mgmt., is (3) : 12331300 .

RICHARD A. (1986). Les populations de Truite de mer (Salmo trutta L.) des mivieres Ome et Touques (Basse-Homandie); Scalimetrie ; Sexage ; Caractéristiques biométriques et démographiques. These Jeme cycle. Fac. Sci. Univ. Rennes, 1 vol.. 54 p.

RICHARD A. (1988). Restauration ces salmonides mignateurs aur la piviere ome: - Ehabilitation des Corges de SaintAubert, définition d'un débit réservé. CSP Rennes, 1 vol., 15 pp.

ROULE L. (1920). Etude sur le saumon des eaux douces de la France considéré du point de vue de son état naturel et du repeuplenent de nos rivières. Finist. Agriculture, Paris, 1 vol., $178 \mathrm{p}$.

SYMONS P.E.K. (1979). Estimated Escapement of Atlantic Salnon (Salmo salar) for naximum snolt production in Rivers of aifferent productivity. J. Fish ries. Boart can., 36 : 132-140.

TELLIER L. (1987). Mise en service d'une station d'étude des migrations des Salmonides migrateurs sur la riviere Elorn (Finistere) : premieres observations sur les populations migrantes d'adultes et de juveniles de Saunon atlantique (Salmo salor L., 1758) Mémoire Jême anněe, ENITEE, 1 vol., 41 p.

THORPE J.E., MORGAN R.I.G., OTTAWAY E.M. (1980). Time of divergence of growth groups between potential $1+$ ande $2+$ smolts among sibling salmon. $j$. Fish Biol., 17 : 13-21.

VIBERT R. (1950). Recherches sur le saumon de I'Adour (Salmo salar L.) (Ages, croissance, cycle génétique, races), 19421948. Anr. St. Cent. Hydro. Aopl., 3 : 27-148. 